\title{
Hybrid strategy for reliable packet transfer and packet loss reduction in cognitive radio Ad-Hoc Network
}

\author{
C.V. Subhaskara Reddy ${ }^{*}$, M.V. Subramanyam ${ }^{2}$ and P. Ramana Reddy ${ }^{3}$ \\ Research Scholar, Department of Electronics and Communications Engineering, JNTUA, Anantapur, India ${ }^{1}$ \\ Professor, Department of Electronics and Communications Engineering, Santhiram Engineering College, Nandyal, \\ Kurnool, A.P, India ${ }^{2}$ \\ Professor, Department of Electronics and Communications Engineering, JNTUA, Anantapur, India ${ }^{3}$
}

Received: 18-July-2021; Revised: 16-November-2021; Accepted: 19-November-2021

(C)2021 C.V. Subhaskara Reddy et al. This is an open access article distributed under the Creative Commons Attribution (CC BY) License, which permits unrestricted use, distribution, and reproduction in any medium, provided the original work is properly cited.

\begin{abstract}
Cognitive radio ad-hoc network is characterized by frequent routing disruptions introduced due to intermittent spectrum availability. Routing disruption increases the packet loss and affects the reliability of the network. This work adopts multiple strategies of reliable link based selective multi path routing, secondary user caching packets, packet redundancy based recovery and adaptive packet generation based congestion control. Recovery is given more importance compared to retransmission in reconstructing the lost packet. Congestion is controlled by applying two strategies of rate control and route participating factor. Reliability of routing is improved by predicting the link failure and handling the failure using selective backup route. With this adoption of multiple strategies, the proposed solution not only minimizes the packet loss but also achieves 3\% higher packet delivery ratio, 9\% lower end-to-end delay and $7.5 \%$ lower communication overhead compared to existing one in cognitive radio ad-hoc network.
\end{abstract}

\section{Keywords}

Cognitive radio Ad-Hoc network, Reliable link routing, Cooperative caching at SU's, Loss recovery.

\section{Introduction}

Cognitive Radio Network is wireless network based on the concept of spectrum sharing for wireless communications. The primary user's spectrum is shared by secondary users when spectrum is not in use by the primary users [1]. Intermittent spectrum availability causes routing disruptions and increases the packet loss in cognitive radio ad-hoc network (CRAHN). Ensuring reduced packet loss especially with multi hop routing scenario is very challenging in CRAHN [2]. Many multi path routing protocols have been proposed with the goal to reduce the packet loss and increase the reliability of CRAHN [3].Same packet is routed in multiple paths, so that the packet can reach the target with higher probability compared to single path routing. But the overhead is higher in conventional multipath routing protocols. This increased overhead contributed to packet loss and congestion in the links. Realizing that, it is difficult to reduce the packet loss with multipath routing alone, this work proposes an integrated strategy to reduce the packet loss in CRAHN [4].

*Author for correspondence 1524
The proposed solution integrates multiple strategies for reducing the packet loss at a reduced network overhead compared to multi path routing protocols. The objective of the proposed work is to reduce the packet loss and improve the reliability of routing in cognitive radio network. Towards this objective, the proposed solution integrates multiple strategies for reducing the packet loss at a reduced network overhead compared to multi path routing protocols. Link failures are predicted in the routing path and selective multipath propagation is proposed to compensate for the link failure. Link failure due to intermittent spectrum availability is modelled considering the idle and busy time distribution of primary user (PU).

The proposed solution adopts two strategies of retransmission and reconstruction to compensate for the packet loss due to unexpected link failures and congestion. Reconstruction is given higher preference compared to retransmission in mitigating packet loss. Sufficient redundancy is built into packet for reconstruction. Cooperative caching at secondary user (SU) is done to avoid the hop count for 
retransmission packets. Uncontrolled packet generation rate without consideration for the network dynamics is an important contributing factor for congestion and packet loss. The proposed solution adjusts the packet generate rate based on cognitive radio network dynamics. A correlation model is built between the packet generation rate and the cognitive radio network dynamics in this work.

Following are the contributions in this work: Link failure prediction model based on PU's idle and busy time distribution, Adaption of packet generation rate based on network dynamics. Selective multipath propagation with backup paths for reducing packet loss, Adaptive retransmission and reconstruction strategy to mitigate packet loss caused due to spectrum uncertainty.

\section{Literature review}

Reducing the packet loss created major attraction for many researchers in recent times. Combining multipath routing with cooperative packet caching offered one such solution for reducing packet loss [5]. Relay nodes cache the packet and rout in alternative paths to reduce packet loss introduced due to routing disruptions. A small buffer is maintained in each node to cache the packet temporarily. On detection of forwarding failure, the cached packets are routed in alternative paths. Multipath routes are found proactively and stored in the nodes. A problem with this approach is that it does not quantify the routes based on reliability and prioritize them for routing. The packet loss in wireless multicast was also reduced by using cooperative communications [6]. On observation of packet loss, a node attempts to recover by cooperating with neighbour nodes. The cooperation happens in level of upstream nodes towards the source. Both packets and routes are cached to speed up loss recovery. The packet has to be cached at multiple places affecting the number of packets to be cache during failures.

An opportunistic routing protocol was proposed to increase the reliability in CRAHN [7]. In this links are scored using reliability metric and this score is used for next hop selection in the routing protocol. The link score is based on the load and it does not account for the spectrum availability. Thus the work can handle only the reliability problems due to loading. The reliability in CRAHN was improved using on demand cluster based routing protocol [8]. Clustering is done based on three criteria of spectrum availability, node stability and power level. A multi objective routing metric is proposed for routing on clustered topology. The routing metric optimizes multiple parameters like delay and delivery ratio. This work does not consider link failure due to spectrum in-availability during routing. A collaborative beam-forming was used along with routing to increase the throughput in CRAHN [9]. SU's collaborated with each other and transmit in regions of primary user's activity by controlling the transmission with beam-forming. The method is highly dependent on placement of nodes. A next hop forwarder selection algorithm was proposed by researchers for cognitive radio networks [10]. The proposed solution is based on geographic opportunistic routing. Channel availability information is known in advance at each link. Based on the channel availability information, hops are selected for forwarding. The method assumes known channel availability slots which are not always true in cognitive radio networks. The geographic routing scheme was further improved for cognitive radio networks with use of network coding [11]. The entire routing path from source to target node is split into multiple small routing segments. The best forwarding set for each routing segment is decided based on availability of spectrum, topology and geometric conditions. The number of transmissions in reduced using network coding. Channel availability information must be known prior for efficient routing in this mechanism.

A spectrum aware routing protocol was introduced to increase the reliability in CRAHN [12]. The optimal forwarding nodes in the routing path are selected based on the link and channel statistics information. In addition to forwarding nodes, this solution also selects the channel to be used for forwarding from among multiple channels. Due to fixing on channel to forward in routing stage, the proposed method cannot adapt to channel dynamics due to spectrum inavailability. Dual stage collaborative spectrum sensing was integrated with opportunistic routing protocol to provide better packet delivery ratio at a lower delay [13]. Two routing metrics were proposed based on spectrum availability to aid in next hop forwarder selection. Throughput in wireless sensor network further improved using heuristic forwarder selection based routing protocol [14]. The forwarding nodes were selected based on the current load handled by the nodes. The solution works for a fixed topology assuming equal distance between the nodes. The work cannot be directly applied for cognitive radio networks as channel state dynamics is not considered. The reliability in cognitive radio 
networks also improved with an opportunistic routing protocol [15].

Geographic location information and spectrum access opportunities are used for best forwarder selection. A new throughput indicator is calculated for each hop based on channel availability, distance to target. Node with higher value for the indicator is used as relay for forwarding the packet. But the method did not solve the link overloading problem. The idea of opportunistic pipeline implemented to improve the reliability in cognitive radio networks [16]. Node outside the main forwarding path participates in the routing opportunistically on observing transmission failure. But without packet caching, the mechanism is not effective against longer duration of failures. The reliability of routing in cognitive radio networks was improved by selecting paths with maximum duration [17]. The algorithm calculates expected link duration and selects the path with maximal expected duration for routing. Expected path duration detailed in this work considers only single channel. The rate in the assigned channel was adapted and routing performed with goal to increase the reliability in CRAHN [18]. The data rate is adjusted based on network state and routing is preferred on interference free links and channels [19]. The reliability in cognitive radio network was improved by applying spectrum aggregation based routing protocol [20, 21]. The work introduced two class of routing with different goals of throughput maximization and delay minimization. Spectrum is aggregated in the MAC layers depending on the routing class opted at the application layer.

A routing protocol based on spectrum availability time was proposed by few researchers for the best performance [22]. But the solution did not consider the backup for packets when spectrum availability prediction becomes erroneous. To estimate the remaining duration of spectrum availability was also proposed based on the expected idle length of channels, sensing period and communication history on the channel [23]. The estimations are used in routing path construction. However, in case of failure there is no backup to recover packet loss. From the survey, the current approaches for reducing the packet loss in cognitive radio network have performance bottleneck due to inaccuracy in failure prediction, adaptive packet flow control during times of failure and high overhead due to multiple path routing. In this work, these challenges are addressed and a hybrid solution to reduce the packet loss is proposed.

\section{Methods}

\subsection{Network model}

Multi hop cognitive radio network model is adopted for this work with existence of multiple PU and SU in the network. Both PU and SU share same set of orthogonal channels. One common control channel is dedicated for SU signalling. Two radios are maintained at each SU - one for switching among orthogonal channels and another for signalling. SU communicate to target node via multi hop routing.

\subsection{Proposed solutions}

The architecture of the solution is given in the below Figure 1.

Following are the important functionalities at each of SU node.

- Reliable routing based on prediction of PU idle/busy time and link failure prediction.

- Loss mitigation through retransmission/reconstruction/re-routing.

- Congestion monitoring and packet rate control.

- Each of the functionalities is explained in detail in the following sections.

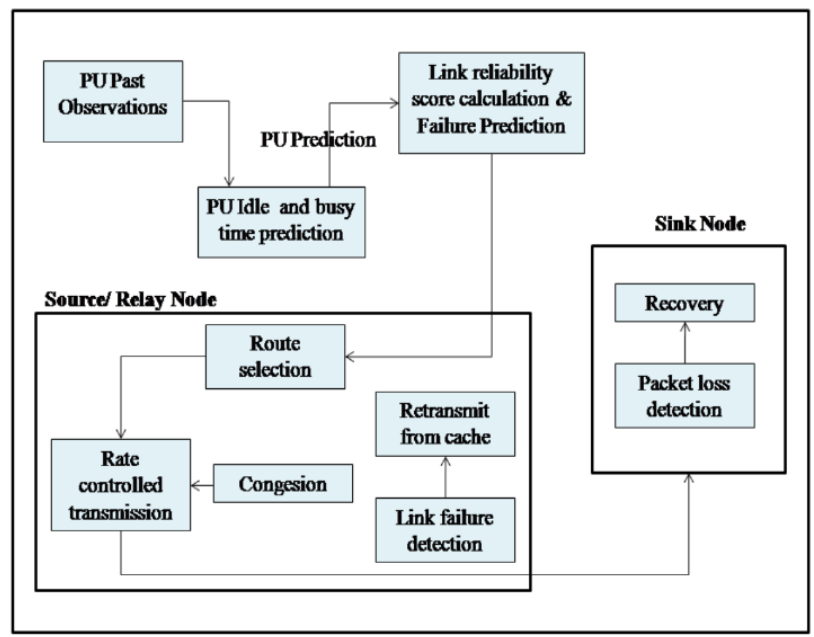

Figure 1 Proposed architecture

\subsection{Reliable routing}

The proposed solution adopts geographic opportunistic routing between the SU's with relay selection based on reliability metric. Each SU calculates its reliability metric based on it observation of PU's idle and busy time, as SU is having least priority in comparison with PU.

The channel status alternates between idle and busy periods. PU is involved in packet transmission during the busy period. On processing of all packets, PU queue becomes empty and its busy period ends. Then 
the idle period start. On arrival of new packet to PU, its idle period ends.

For packet arrival rate of $\gamma$ packets/sec according to poison process, the primary user encounters with exponential distribution of idle period which is represented by the termIp. The Laplace transform $\mathrm{Ip}(\mathrm{s})$ is represented in Equation 1.

$I_{p}(s)=E\left(e^{-s I_{p}}\right)=\int_{0}^{\infty} e^{-s t} \gamma e^{-\gamma t} d t$

$I_{p}(s)=\frac{\gamma}{s+\gamma}$

The primary user keeps transmitting units until its queue is empty. Transmission errors can result in retransmission of packet till packet is received successfully. Packet service time depends on the time taken as well as successive numbers of packet transmission. The successive numbers can be calculated as per Equation 2.

$$
\begin{aligned}
P_{r}(N=n)=\left(q_{1} q_{2}\right)^{n-1} p_{1} & +U(n)(1 \\
& \left.-q_{1} q_{3}\right)\left(q_{1}^{n-1} \sum_{i+j=n-2} q_{2}^{i} q_{3}^{j}\right) p_{2}
\end{aligned}
$$

Where

$U(n)=\left\{\begin{array}{l}0, n=1 \\ 1, n>1\end{array}\right.$

In the above equation, the successive numbers of transmission are represented with the term $\mathrm{N}$ and $q_{i}$ is calculated asper Equation 3.

$q_{i}=1-p_{i}$

Where $p_{i}$ is the probability of transmitting the packet successfully during the time $\mathrm{i}$.

Based on the packet transmission time $l_{p}$ and numbers $\mathrm{n}$, the Laplace transform of service time of the PU is calculated asper Equation 4.

$M_{p}(s \mid N=n)=$

$\int_{0}^{\infty} M_{p}\left(s \mid L_{p}=l_{p}, N=n\right) f_{L_{p}}\left(l_{p}\right) d l_{p}=$

$\int_{0}^{\infty} e^{-s n l_{p}} f_{L_{p}}\left(l_{p}\right) d l_{p}$

Where $f_{L_{p}}$ is the probability density function of packet transmission time and $L_{p}$ is its Laplace transform. Applying the rule of packet service time [24], the mean busy period is calculated as per Equation 5.

$B_{p}=\frac{M_{p}}{1-\rho_{p}}$

Where $M_{p}$ is the mean service and $\rho_{p}$ is the traffic load of the primary user. Based on it, the SU calculates its reliability score as Equation 6 . $r s=I_{p}+1 / B_{p}$

A SU which needs to find the next hop node, send a HELLO message to all the neighbouring SU's over the control channel, each SU receiving the HELLO message, sends HELLO_RES with the calculated reliability score. The SU selects the next hop SU with the highest reliability score as the best relay for forwarding the packet.

\subsection{Packet loss mitigation}

The packet loss is mitigated in the proposed work through two methods of

1. Retransmission

2. Recovery

The node failure, link quality degradation and spectrum unavailability can cause failures in cognitive radio networks.

The failure due to spectrum unavailability can be detected by energy based spectrum sensing at the SU node. The spectrum sensing is a binary hypothesis test given can be calculated using Equation 7 .

$H_{0}: y_{i}(t)=\eta_{i}(t)$, whenPUisabsent

$H_{1}: y_{i}(t)=h_{i} x(t)+\eta_{i}(t)$, whenPUispresent

Where $\mathrm{t}=1,2, \ldots N_{x}$ is the sample space, $N_{x}$ is the total number of samples in received signal and calculated as per Equation 8.

$N_{x}=2 B T_{s}$

The predefined bandwidth andsensing time can be represented by the notations $\mathrm{B}$ and $T_{S}$ similarly $y_{i}(t)$ would be the received signal at $\mathrm{SU}$. The transmitted signal at PU is represented as $\mathrm{x}(\mathrm{t})$ and it has been treated as Gaussian Random Process where variance and mean value is zero. $\eta_{i}(t)$ is $i^{\text {th }}$ channel Additive White Gaussian noise. The channel gain is represented by the term $h_{i}$ which would be constant over every sensing period.

The failure due to node failure or link quality degradation can be found using Hello based approach. Periodic Hello beacons are sent from the SU node to its neighbours. Link is active (up) on reception of certain number of successive Hello beacons and it can be used for routing. Otherwise, SU considers the link as inactive (down) and leaves it.

The maximum delay $(\partial)$ to model the link failures depends on the Hello interval $\left(T_{B}\right)$ and number of missing Hello (K). It is calculated as per Equation 9. 
$\partial=K T_{B}+\epsilon$

$\partial$ has the uniform distribution on

$\left[(K-1) T_{B}, K T_{B}\right]$

and the average value $\left(\partial^{\prime}\right)$ is given as

$\partial^{\prime}=\frac{(2 K-1) T_{B}}{2}$

Where $\mathrm{K} \geq 1$ and $T_{B}>0$. On detection of failure at an $\mathrm{SU}$, mitigation using recovery is first attempted and if not retransmission is attempted.

Erasure coding (n, $\mathrm{k})$ is used to split the contents into $\mathrm{n}$ packets. Out of these $\mathrm{n}$ packets, only $\mathrm{k}$ minimal packet is needed for reconstruction of the content. All $\mathrm{n}$ packets are sent over network. There can $\mathrm{n}-\mathrm{k}$ packets dropped. Even if n-k packets are dropped, the original content can be reconstructed without need for retransmission.

Reed Solomon erasure coding is used in this work. If $\mathrm{SU}$ finds that at least $\mathrm{k}$ packet of $\mathrm{n}$ has been sent successfully, it does not move to retransmission mode. Instead, it relies on the target or sink node to reconstruct the packet using $\mathrm{k}$ received packets. In case SU finds that at least $\mathrm{k}$ packet of $\mathrm{n}$ has not been sent successfully, it attempts retransmission.

Each SU have a buffer to cache the transmitted packets and on observation of failure in the upstream node, reattempts via different relay apart from the relay in routing path. The number of backup relay node used for forwarding is decided dynamically based on channel outrages.

Say there are $\mathrm{N}$ relays and these $\mathrm{N}$ relays will be used when there is a packet outrage probability of 1 . From this, the number of relays $N_{r}$ to be selected is given as per Equation 10.

$N_{r}=N /\left[1-\exp \left(-\frac{\beta N_{o} d^{\gamma}}{G}\right)\right]$

$\beta$ is constant set as 0.5 in this work.

\subsection{Congestion monitoring and control}

Congestions can cause packet loss and this work adopts adaptive packet rate generation to mitigate the congestion. The round trip time (RTT) is used as metric for measuring congestion. The RTT is modelled as probability mass function of delay distribution is given in Equation 11.

$$
R T T=\left\{\begin{array}{c}
\sum_{i=0}^{\infty} f_{i}(a) \cdot f_{i}(b), x=0 \\
\sum_{i=0}^{\infty} f_{i}(a) \cdot f_{2 x+i}(b)+\sum_{i=0}^{\infty} f_{i}(b) \cdot f_{2 x+i}(a), x>0
\end{array}\right.
$$

Forward direction from transmitter to receiver is denoted as ' $a$ '. Backward direction from receiver to transmitter is denoted as ' $b$ '. The probability mass function of delay in a direction $\mathrm{z}$ is denoted as $\mathrm{f}(\mathrm{z})$.

Based on the RTT value, the number of packets of node to be sent from SU node $N_{p}$ is calculated as in Equation 12.

$N_{p}=\frac{N * K}{R T T} *\left(1-P_{o}\right)$

Where $\mathrm{K}$ is the minimum value for RTT and $\mathrm{N}$ is maximum number of packets that can be sent from $\mathrm{SU}$ and $P_{o}$ is the packet outrage probability. By this way flow rate is controlled in the network thereby mitigating the probability of congestion.

The pseudo code of the overall algorithm for routing at source node is given below

Algorithm: Reliable_Route

Input: src node, dest node, payload

Shares $\leftarrow$ Erasure_coding(payload,n,k);

For $\mathrm{i}=1: \mathrm{n}$

Packet.src $=$ srcnode;

Packet.dest $=$ dest_node;

Packet.payload = Shares[i];

neighbours

Res[] $\leftarrow$ Send Hello broadcast to all

Maxscore $\leftarrow 0$

Whomax $\leftarrow-1$

For all Hello_Res in Res

If Hello_Res.rel_score> Maxscore

Maxscore $\leftarrow$ Hello_Res.rel_score Whomax $\leftarrow$ Hello_Res.src;

End

End

Forward Packet to Whomax

end

3.6The proposed solution has following novelties Laplace transform based accurate link failure prediction model and routing decision based on link failure probability. Network dynamics based adaptive packet flow control at source and at relay nodes. Low overhead selective multipath propagation with backup near the packet failure links to reduce packet loss. Packet loss reduction integrating adaptive retransmission and reconstruction with first priority for reconstruction and in failure of it adaptive retransmission is initiated. 


\section{Results and discussions}

The proposed routing solution was simulated in NS2. The parameters considered to conduct the simulation is tabulated in Table 1. Performance comparison is done against following solutions. Spectrum aware routing algorithm by Tran-Dang and Kim [23] and Spectrum availability routing by Salameh and Khasawneh [22]. The performance is compared in terms of Packet success ratio, Average latency and Network overhead.

Table 1 NS2 Simulation parameters

\begin{tabular}{lll}
\hline Parameters & Values & Unit \\
\hline No. of channels & 4 & - \\
\hline Channel available probability & $\{0.7,0.3,0.6,0.8\}$ & - \\
\hline No. of PUs per channel & 16 & - \\
\hline PU transmission range & 250 & $\mathrm{M}$ \\
\hline Number of CR nodes & 100 to 400 & - \\
\hline CR node data rate & 2 & $\mathrm{Kbps}$ \\
\hline Buffer size & 8 & $\mathrm{~Kb}$ \\
\hline Sensing time & 1 & $\mathrm{Ms}$ \\
\hline Channel switching time & 1 & $\mathrm{Ms}$ \\
\hline Simulation time & 10 & Minutes \\
\hline
\end{tabular}

The packet success ratio is measured by varying the percentage of nodes generating the packets to sink for $100 \mathrm{CR}$ nodes in the network. Packets are sent from certain designated nodes to the sink node and packet success ratio is measured by the number of packets received at sink. The result is plotted as per the data in Figure 2.

The packet success ratio in the proposed solution is on average $6 \%$ more compared to Tran-Dang and Kim [23] and 4\% more compared to Salameh and Khasawne [22]. The integrated strategy of reliable route selection, packet caching for retransmission and flow control has increased the packet delivery ratio in the proposed solution. By fixing the number of packet generation nodes as $10 \%$, packet success ratio is measured for different number of CR nodes. The result is plotted as per the data in Figure 3. The packet success ratio drops with increase in number of $\mathrm{CR}$ nodes. This drop is due to more $\mathrm{CR}$ nodes competing for the spectrum. The packet success ratio in the proposed solution is on average 3\% more compared to both solutions of Tran-Dang and Kim [23] and Salameh and Khasawne [22]. Retransmission and recovery strategy in the proposed solution ensure lower packet loss in the proposed solution compared to existing works.

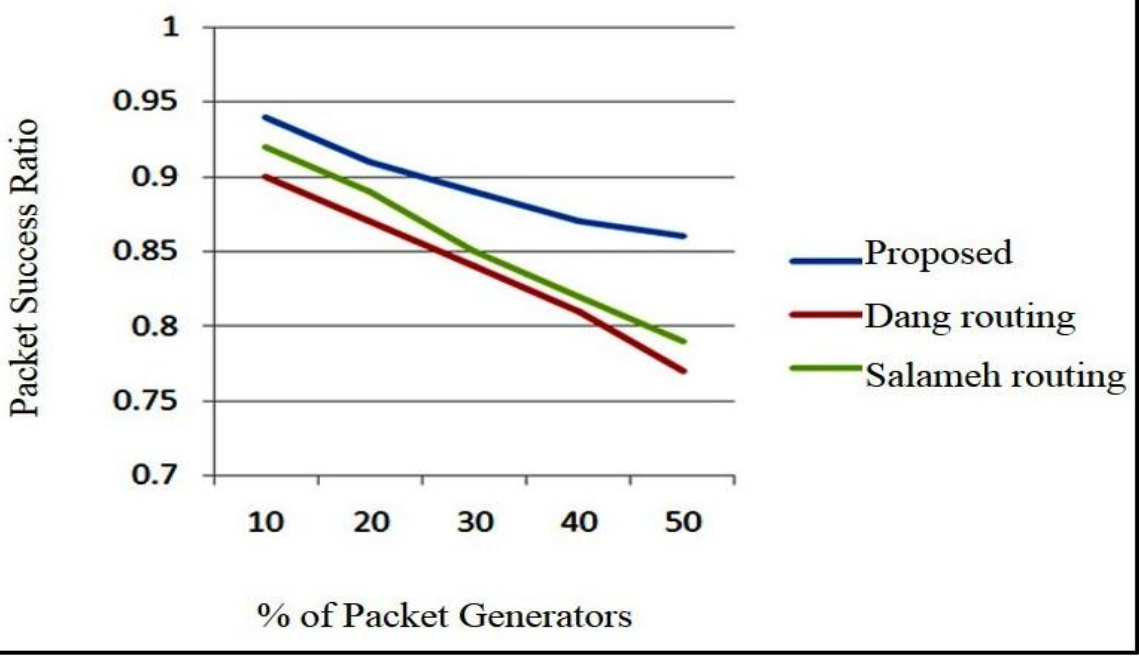

Figure 2 Packet success ratio with varying $\%$ of packet generators 


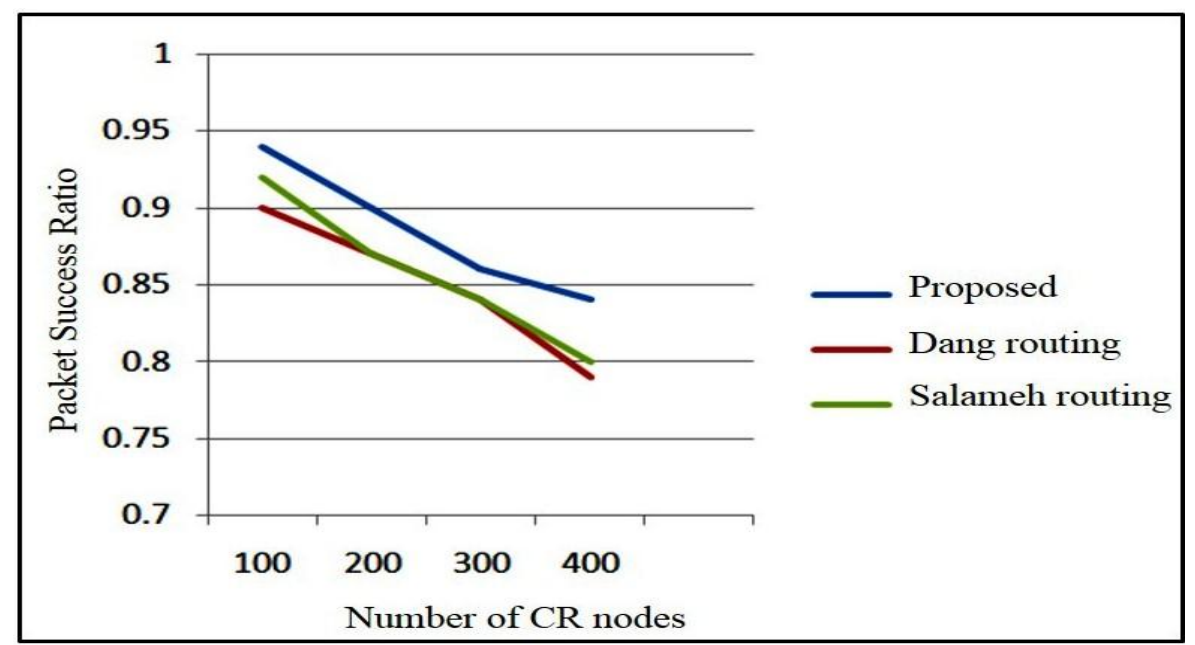

Figure 3 Packet Success Ratio over CR nodes

Keeping the number of CR nodes as 100 and a constant packet generation rate of $10 \%$ for simulation duration of 10 minutes, packet success ratio is measured as per the data in Figure 4(a). The packet success ratio increased over the simulation time. But the packet success ratio is on average $8 \%$ more compared to Tran-Dang and Kim [23] and 7\% more compared to Salameh and Khasawne [22]. Compared to existing works, the proposed solution has higher packet delivery ratio, due to use of adaptive flow control strategy.

The average latency for packet arrival at sink is measured for varied number of CR nodes at $10 \%$ packet generation rate and the result is taken as per the data in Figure $4($ b).

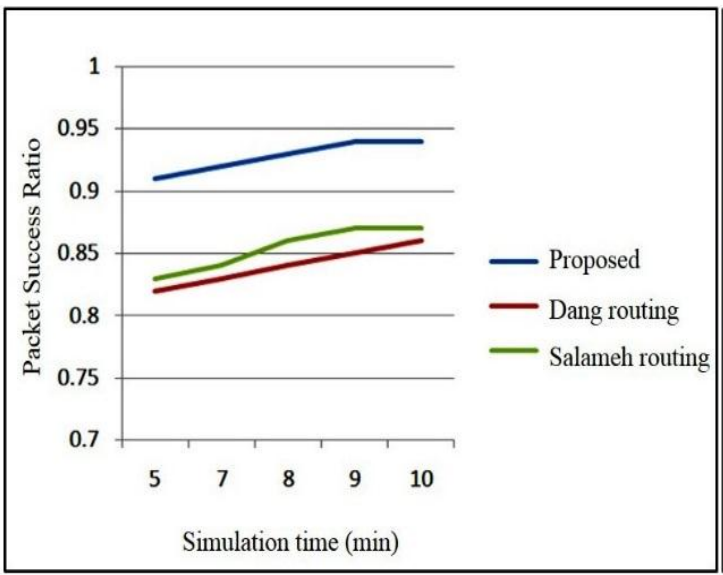

(a)

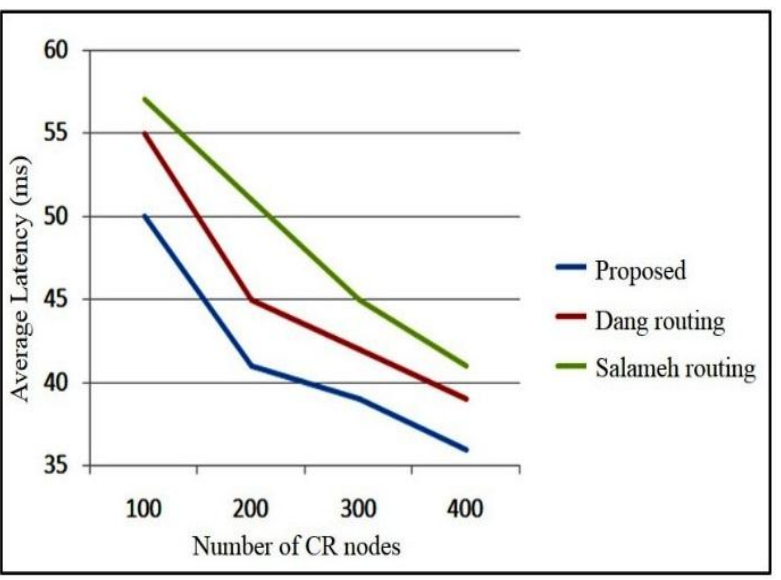

(b)

Figure 4 (a) Packet success ratio over time (b) Average latency

The end-to-end delay is on average 9\% lower in the proposed solution compared to Tran-Dang and Kim [23] and $16.87 \%$ lower compared to Salameh and Khasawne [22]. The use of geographic opportunistic routing and only selective multi path propagation has reduced the end-to-end delay in the proposed solution compared to existing works. The communication overhead is measured in terms of volume of data packets apart from data payload. It is measured by 1530 varying the number of $\mathrm{CR}$ nodes and the result are taken as per the data in illustrated in Figure 5. The communication overhead is on average $7.5 \%$ lower in the proposed solution compared to Tran-Dang and Kim [23] and 5.4\% lower compared to Salameh and Khasawne [22]. The use of geographic routing has reduced the communication overhead in the proposed solution. A complete list of abbreviations is shown in Appendix I. 


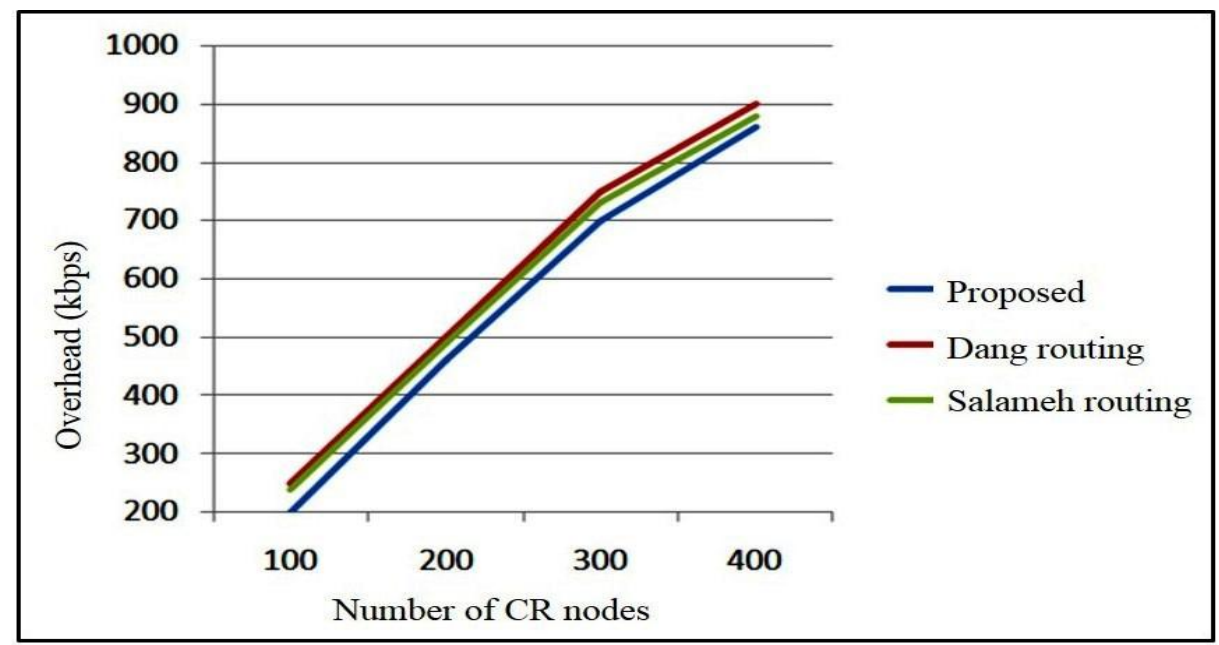

Figure 5 Communication overhead

\section{Conclusion and future work}

In this work, we considered the problem of reducing the packet loss and increasing the packet success ratio in the cognitive radio network.

An integrated solution is proposed combining reliability routing estimating the link failure and handling with selective back up route, mitigating the packet loss using retransmission /reconstruction/rerouting and congestion monitoring by the adoption of packet rate control. Through simulation, it is observed that proposed solution has 3\% higher packet delivery ratio, 9\% lower end-to-end delay, $7.5 \%$ lower communication overhead compared to existing solution. The higher packet delivery with lower delay and network overhead is achieved for cognitive radio ad-hoc network.

False sensing observation when channel is busy and energy consumption at relay nodes are two limitations in this work. False sensing will results into link failure because of spectrum uncertainty and leads to packet loss. Less energy consumption nodes doesn't break up frequently and can handle with spectrum mobility as well packet loss.

Future availability of present channel and its impact on packet loss recovery is one major challenge that researcher desires to carry as future scope of the work.

\section{Acknowledgment}

None.

\section{Conflicts of interest}

The authors have no conflicts of interest to declare.

\section{References}

[1] Moghaddam SS. Cognitive radio in $4 \mathrm{G} / 5 \mathrm{G}$ wireless communication systems. BoD-Books on Demand; 2018.

[2] Lin P, Jia J, Zhang Q, Hamdi M. Dynamic spectrum sharing with multiple primary and secondary users. IEEE Transactions on Vehicular Technology. 2011; 60(4):1756-65.

[3] Kamruzzaman SM, Fernando X, Jaseemuddin M. Energy aware multipath routing protocol for cognitive radio ad hoc networks. International Journal of Communication Networks and Information Security. 2016; 8(3):187-96.

[4] Singh K, Moh S. An energy-efficient and robust multipath routing protocol for cognitive radio ad hoc networks. Sensors. 2017; 17(9):1-17.

[5] Kim S, Kim C, Jung K. Cooperative multipath routing with path bridging in wireless sensor network toward IoTs service. Ad Hoc Networks. 2020.

[6] Krishnan CG, Robinson YH, Julie EG, Bamini AA, Kumar R, Thong PH. Hybrid cache management in ad hoc networks. Wireless Personal Communications. 2021; 118(4):2843-65.

[7] Duan W, Tang X, Zhou J, Wang J, Zhou G. Load balancing opportunistic routing for cognitive radio ad hoc networks. Wireless Communications and Mobile Computing. 2018.

[8] Zareei M, Mohamed EM, Anisi MH, Rosales CV, Tsukamoto K, Khan MK. On-demand hybrid routing for cognitive radio ad-hoc network. IEEE Access. 2016; 4:8294-302.

[9] Guirguis A, Karmoose M, Habak K, El-Nainay M, Youssef M. Cooperation-based multi-hop routing protocol for cognitive radio networks. Journal of Network and Computer Applications. 2018; 110:2742.

[10] Tang X, Chang Y, Zhou K. Geographical opportunistic routing in dynamic multi-hop cognitive radio networks. In computing, communications and applications conference 2012 (pp. 256-61). IEEE. 
[11] Tang X, Zhou J, Xiong S, Wang J, Zhou K. Geographic segmented opportunistic routing in cognitive radio ad hoc networks using network coding. IEEE Access. 2018; 6:62766-83.

[12] Wang J, Yue H, Hai L, Fang Y. Spectrum-aware anypath routing in multi-hop cognitive radio networks. IEEE Transactions on Mobile Computing. 2016; 16(4):1176-87.

[13] Cui C, Man H, Wang Y, Liu S. Optimal cooperative spectrum aware opportunistic routing in cognitive radio ad hoc networks. Wireless Personal Communications. 2016; 91(1):101-18.

[14] Zikria YB, Nosheen S, Choi JG, Kim SW. Heuristic approach to select opportunistic routing forwarders (HASORF) to enhance throughput for wireless sensor networks. Journal of Sensors. 2015.

[15] Liu Y, Cai LX, Shen XS. Spectrum-aware opportunistic routing in multi-hop cognitive radio networks. IEEE Journal on Selected Areas in Communications. 2012; 30(10):1958-68.

[16] Liu A, Chen W, Liu X. Delay optimal opportunistic pipeline routing scheme for cognitive radio sensor networks. International Journal of Distributed Sensor Networks. 2018; 14(4):1-13.

[17] Liu J, Ren P, Xue S, Chen H. Expected path duration maximized routing algorithm in CR-VANETs. In international conference on communications in China 2012 (pp. 659-63). IEEE.

[18] Tang F, Li J. Joint rate adaptation, channel assignment and routing to maximize social welfare in multi-hop cognitive radio networks. IEEE Transactions on Wireless Communications. 2016; 16(4):2097-110.

[19] Ping S, Aijaz A, Holland O, Aghvami AH. SACRP: a spectrum aggregation-based cooperative routing protocol for cognitive radio ad-hoc networks. IEEE Transactions on Communications. 2015; 63(6): 201530.

[20] Reddy CS, Subramanyam MV, Reddy PR. Implementation and performance evaluation of various multicast routing protocols in cognitive radio ad-hoc network: a performance review. In international conference on wireless communications, signal processing and networking 2017 (pp. 2194200). IEEE.

[21] Reddy CS. A lecture notes on cognitive-based radio ad hoc networks. In proceedings of 2 nd international conference on micro-electronics, electromagnetics and telecommunications 2018 (pp. 647-55). Springer, Singapore.

[22] Salameh HB, Khasawneh H. Routing in cognitive radio networks with full-duplex capability under dynamically varying spectrum availability. ICT Express. 2021; 7(1):115-20.

[23] Tran-dang H, Kim DS. Link-delay and spectrumavailability aware routing in cognitive sensor networks. IET Communications. 2020; 14(20):363951.

[24] Kurth M, Zubow A, Redlich JP. Multi-channel linklevel measurements in 802.11 mesh networks. In proceedings of the international conference on wireless communications and mobile computing 2006 (pp. 937-44)

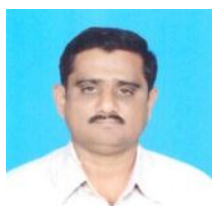

Mr. C. V. Subhaskara Reddy presently pursuing Ph.D from JNTUA, Ananthapur and his field of Interest includes computer networks, router designing, bio-medical engineering and signal processing etc. He is working as Associate Professor in ECE, Dept. of Nandyal. ECE, Santhiram Engineering College,

Email: subaskar.reddy@gmail.com

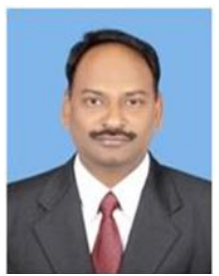

Dr. M. V. Subramanyam awarded Ph.D from JNTU, Hyderabad in 2007 in the area of Ad-Hoc Wireless Networks. He Published 162 research papers and guided 15 research scholars for their PhD award. Presently, he is working as Professor of ECE and Principal of Santhiram Engineering College, Nandyal, Kurnool, A.P, India

Email: principal.srec.x5@gmail.com

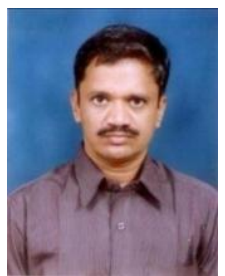

Dr. P. Ramana Reddy, completed Ph.Dfrom JNTUniversity, Ananthapur in the field of Image Processing. Presently he is working as Professor in ECE Department, JNTUA, College of Engineering, Ananthapur. His research interests include Signal, Image, Video and VLSI Signal processing. He published 90 research papers and guided 09 research scholars.

Email: prrjntu@gmail.com

Appendix I

\begin{tabular}{lll}
\hline S. No. & Abbreviation & Description \\
\hline 1 & CR & Cognitive Radio \\
\hline 2 & CRAHN & Cognitive Radio Ad-hoc Network \\
\hline 3 & PU & Primary User \\
\hline 4 & RTT & Round Trip Time \\
\hline 5 & SU & Secondary User \\
\hline
\end{tabular}

